\title{
On one approach to the detection of infrasonic signals of irregular natural phenomena in the instrumental observations time series at the student interdisciplinary testing ground
}

\author{
Nikolay Kudryavtsev*, Varvara Safonova, and Albina Temerbekova \\ Institute of Physics \& Mathematics and Engineering \& Technology, Gorno-Altaisk State University \\ (GASU), 649000 Gorno-Altaisk, Russia
}

\begin{abstract}
The work is devoted to the analysis of time series and the problem of processing signals obtained as a result of the design approach implementation during the organization of instrumental observations of irregular natural phenomena at the student interdisciplinary testing ground. The objective of the work is to study the methods of processing noisy signals obtained as a result of monitoring the infrasonic environment, which make it possible to automate the search for fragments of the time series generated by irregular natural phenomena. At the beginning of the work, a brief explanation of the essence of the measuring scientific experiment carried out within the framework of the project approach used in the additional education of students and schoolchildren shall be given. The following is a review of publications describing various approaches to the analysis of nonstationary time series obtained in the process of instrumental observations. As the main method of time series analysis, it is proposed to use the algorithm for calculating the fractal dimension of the time series, proposed by T. Higuchi [1]. During studying of the time series of infrasonic signals, a number of regularities were discovered that contribute to the development of an original procedure for processing and transforming the signal under study, which makes it possible to determine the time intervals of fragments of the time series corresponding to the signals of the desired natural phenomena. The essence of the proposed approach lies in the preliminary preparation of the time series by processing the data with a simple normalized difference filter, previously smoothed by performing the coenvolution (convolution) operation with a Gaussian kernel; determining the step of segmenting the normalized time series, calculating fractal dimensions and averaged amplitudes for each of the segments of the time series and obtaining on their basis vectors of changes in dimensions and amplitudes with their subsequent element-wise multiplication. It is shown that the maximum values of the components of the resulting vector are indicators of timestamps for the location of the desired signals.
\end{abstract}

${ }^{*}$ Corresponding author: ngkudr@mail.ru 


\section{Introduction}

As a structural basis (framework) of the educational process in the system of engineering education for many years, a project approach is used all over the world. In the Western education system, for example, the term project-led education (PLE) is often used. It is aimed at developing the skills of studying and solving large-scale complex problems in conditions of the need to search for reliable information and teamwork [2]. PLE is based on student team activity, which includes, along with training, the solution of fairly serious open source projects. Not only abroad, but also in Russia, one can find examples in which both individual technical solutions and whole complex infrastructure projects are implemented as educational projects. In the scientific work [3], for example, the problem of creation and the necessity of existence in the Moscow region of various geo-polygons is considered: educational, scientific, production, demonstration, test, measurement, observation. The work notes that such polygons are simply necessary for carrying out practices of various topics: geodetic, topographic, geophysical, geographical, geological, geomorphological, geobotanical, hydrological, meteorological, landscape, astronomical, forest inventory, smart farming, etc. The work [4] also explores the idea of linking an effective educational process in the field of physical geography and a project to create a kind of meteorological testing ground in the form of a network of simple meteorological stations. Indicative is the fact that the authors of a number of works used independentlymade measuring devices based on a Raspberry Pi mini-computer as measuring equipment, and IoT (Internet of Things) technologies were used to transfer, store and monitor data [5, 6]. One of the variations of the design approach used in the educational process is the design interfaces method, the use of which made it possible to simplify the creation of a number of projects described in this work [7].

This paper describes the results obtained in the development of one of the design modules of the student interdisciplinary testing ground [8], deployed on the basis of the Institute of Physics \& Mathematics and Engineering \& Technology of Gorno-Altaisk State University - a data processing and analysis module. In the process of studying the problem of processing signals obtained as a result of the implementation of the design approach when organizing instrumental observations of irregular natural phenomena by means of infrasound monitoring, the authors analyzed a number of works in which similar problems were solved.

So in the article [1] by the author T. Higuchi, a new method for measuring the fractal dimension of the set of points $(t, f(t))$, forming the graph of the function $f$ defined on a unit interval, is presented. First, the method was applied to the fractional Brownian function, which has the property of similarity of mixing for all scales. As a result, the author managed to obtain a stable and sharp fractal dimension. This method is also applied to observations of natural phenomena. It was noted in the work that the value of the fractal dimension can change continuously in some cases and abruptly in a critical time scale under other conditions. The author notes that the method proposed by him makes it possible to reveal unusual natural phenomena with an abrupt change in the fractal dimension of the signals under study. This property of fractal dimensions, applied to the segments of the investigated time series, made it possible to partially solve the problem set to us.

Authors of work [9] F. Cervantes-De la Torre, J. I. Gonzalez-Trejo, C. A. Real-Ramirez and L. F. Hoyos-Reyes note that fractal dimension is an important characteristic of the studied systems, since it contains information about their geometric structure through the several scales. When analyzing nonlinear time series, they used three algorithms: spectral analysis, altered range analysis, and Higuchi's algorithm. The analyzed time series were associated with natural phenomena and demonstrated behavior depending on the time scale 
of measurements. The authors especially noted that the fractal dimension, calculated using the Higuchi method, turned out to be constant at all time scales.

In article [10], the author of Sladjana Spasic proposed a new numerical method for calculating the two-dimensional fractal dimension of the surface. This method is a generalization of the Higuchi method for calculating the fractal dimension of a plane curve. According to the author, the Higuchi method is much simpler than other similar methods, since it allows to analyze images quickly and can be used alone or in combination with other methods. The Higuchi method has an advantage over other fractal methods when it comes to speed, accuracy, simplicity, and analysis time.

In article [11], the author Christoph Bandt introduces a number of new methods of time series analysis that only use the comparison of values, but not their actual value. Since the introduced methods use only the order of values, they are resistant to nonlinear signal distortions, and the required parameters are calculated very quickly. The local and global order structure is defined in terms of matrices or rank numbers. Local ranks, Kendall correlation coefficient and permutation entropy, as a measure of complexity, are introduced by the author in such a way that they contain a scale parameter that allows studying time series at different scaling options.

In their article, Christoph Bandt and Bernd Pompe [12] introduce time series complexity parameters based on the comparison of adjacent values. This definition applies directly to arbitrary data in the real world. For some well-known chaotic dynamical systems, it is shown that the introduced complexity parameters behave similarly to the Lyapunov exponents. The permutation entropy seems to be a particularly suitable measure of complexity for chaotic time series, in particular, in the presence of dynamic and observational noise. The advantages of the considered method, according to the authors, are its simplicity, extremely fast calculation, reliability and invariance with respect to nonlinear monotonic transformations.

The fractal dimension of a waveform is a powerful tool for detecting transients. In particular, during analyzing electroencephalograms and electrocardiograms, this feature was used to identify and distinguish between specific states of physiological functions. In [13], the authors of Rosana Esteller, George Vachtsevanos, Javier Echauz and Brian Litt analyze and compare the most common methods for assessing the fractal dimension of biomedical signals in the time domain (when considering the time series as a geometric object). The research results presented in the article show that for specific applications a careful choice of the fractal dimension algorithm is required.

In [14], the authors performed a non-stationary analysis of fractal dimension in relation to a seismic signal. The authors also calculated the temporal evolution of the fractal dimension using the Higuchi algorithm, with $\mathrm{k}=6$ and $\mathrm{N}=90,000$ samples (30 minutes) without overlapping windows. The results of this study highlight that medium-term seismic precursors of paroxysmal activity provide valuable data for the development of an early warning system for paroxysmal explosions based on strain gauge measurements.

In [15], the authors presented the results of a multifractal analysis of meteorological time series received from four stations in Poland and Bulgaria located in different climatic zones. The authors are confident that in addition to the commonly used procedures based on quantitative assessment of trends and fluctuations of the corresponding meteorological quantities, it is necessary to use more advanced methods, including fractal analysis and chaos theory, to understand the dynamics of processes occurring in individual layers of the atmosphere. These new approaches allow not only to deduce the trend and seasonality within the analyzed time series, but also to evaluate other features, such as long-range power-law correlations, which means that the decay of these correlations occurs in accordance with a power law, and not as a more intuitive exponential decay. 
The article [16] proposes a new technique for signal-to-noise identification and targeted suppression of magnetotelluric signals. This method is based on the fractal-entropy clustering algorithm, which automatically identifies signal areas damaged by common noise (square, triangular, and impulse waves), providing targeted noise suppression and preventing the loss of useful information during filtering. To implement the procedure, four characteristic parameters are used - the cellular method for calculating the fractal dimension, the Higuchi fractal dimension, fuzzy entropy, and approximate entropy. Compared to the traditional noise cancellation strategy, which 'blindly' applies a filter to the entire data set, the proposed method can automatically identify and purposefully suppress intermittent noise in the signal. The resulting resistance-phase curve is more continuous and smoother, and the tendency of a slow change in the low-frequency range is maintained more accurately.

The article [17] describes the application of a two-stage computational methodology of five different methods for analyzing evolving chaos (DFA, analysis of fractal dimension using the Higuchi, Katz and Sevchik methods and power analysis). All analysis methods showed results that exceeded the threshold values for the data. Numerous stable segments were found with DFA values between $1.6 \leq \alpha \leq 2.0$, fractal dimension from $1.4 \leq \mathrm{D} \leq 2.0$, and exponential exponent from $2.2 \leq \beta \leq 3.0$. Activity has also been reported in identical time windows between or after these earthquakes.

The works considered above confirm that both the fractal dimension calculated using the Higuchi algorithm and its dynamic change in time can serve as good parameters characterizing the dynamics of the studied time series.

The purpose of this work is to study the possibility of using the fractal dimension of time series, calculated using the Higuchi algorithm when processing the results of instrumental measurements and automating the procedure for detecting signals generated by irregular natural phenomena.

\section{Equipment and research methods}

The work presents the results of studies of time series, which are infrasonic signals, digitized using the equipment developed in the laboratory of robotics GASU [8]. To conduct infrasonic instrumental monitoring, several infrasonic sensors were used with a sensitivity of the order of $0.025 \mathrm{~Pa}$ at frequencies from $0.1 \mathrm{~Hz}$ to $1 \mathrm{~Hz}$ and $0.01 \mathrm{~Pa}$ at frequencies from $1 \mathrm{~Hz}$ to $5 \mathrm{~Hz}$, developed on the basis of specially selected electrets microphones, the signal from which was amplified by an original module containing a high-impedance repeater on an operational amplifier, a signal amplifier and an active lowpass filter with a cutoff frequency of the order of $5 \mathrm{~Hz}$. In addition, the sensors were installed at spatially separated measuring points of the polygon in conjunction with ultralow-frequency resonators of various designs.

Measurements using the sensors listed above were carried out under the control of Atmega328 microcontrollers (Arduino Nano platform). All data were digitized using 16-bit analog-to-digital converters with a sampling rate of about $10-12 \mathrm{~Hz}$ and recorded via a galvanically isolated serial connection into asynchronous dataloggers with external quartz frequency stabilization, equipped with a real-time clock and having a special correction function implemented using a GPS receiver with an accurate 1pps timing signal. Data loggers were developed on the basis of the PIC24FJ64GA102 microcontroller in various designs and showed fairly good reliability characteristics over a long period of operation. It should be noted that meters and data loggers are practically autonomous measuring systems, limited in our case only by the presence of an external power supply and the volume of the used SD\MMC card. 
Several functions have been written in Python language to carry out computational experiments. The listing of the function $\operatorname{Lx}(\mathrm{x}, \mathrm{k})$ for calculating the length of the curve $\mathrm{L}(\mathrm{k})$ depending on the time interval $\mathrm{k}$ by the Higuchi method is presented below.

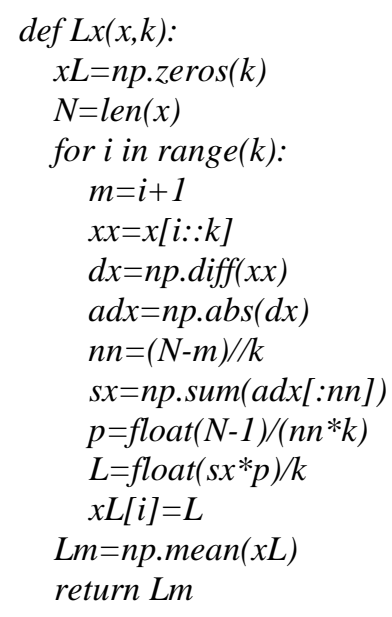

Also, a function was developed to calculate the actual fractal dimension HigFunc(xx1). In addition to the sought-for value of the fractal dimension, this function returns the vectors of k-values (time intervals) was taken the logarithm by base 2 and by base of the lengths of the curves $\mathrm{L}(\mathrm{k})$.

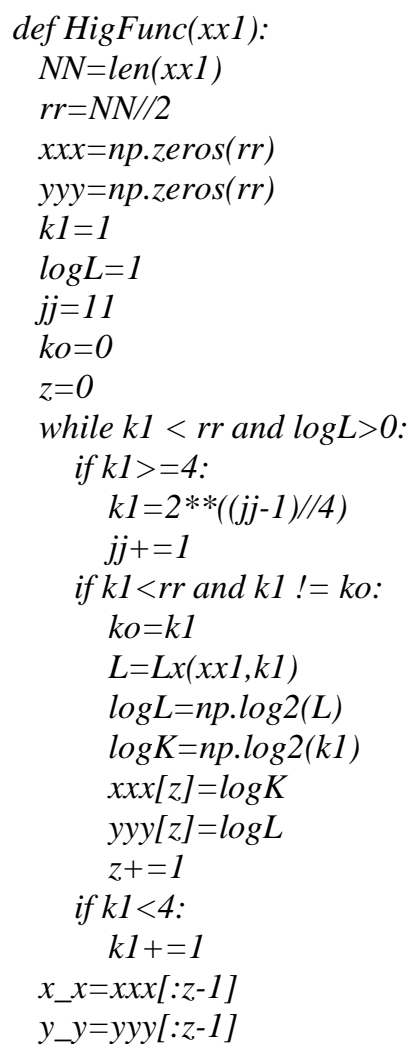




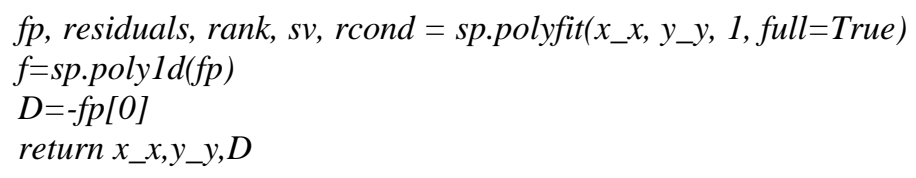

In addition to the aforementioned functions, a time series smoothing scheme was implemented by processing the data with a simple normalized difference filter, preprocessed by performing a coenvolution (convolution) operation with a Gaussian kernel having a size of 25 samples and a standard deviation of 3 .

\section{Results}

Before proceeding with the study of infrasonic signals, it was decided to use the recommendation of the author of the article [1] and reduce the number of investigated time intervals $\mathrm{k}$. It was also decided to exclude from consideration those time intervals $\mathrm{k}$ for which the values of $\log 2(\mathrm{~L}(\mathrm{k}))$ took values less than zero.

Before starting computational experiments with fractal dimension, a test set $\mathrm{Y}$ (i) (i= $1,2, \ldots, N$ ) was generated by performing for each Y (i) summation of $1000+i$ normally distributed numbers with zero expectation function and standard deviation equal to 1 . $\mathrm{N}=$ $2^{17}$ was chosen based on the data of the example from the article [1]. The generation procedure was implemented in Python language and looked:

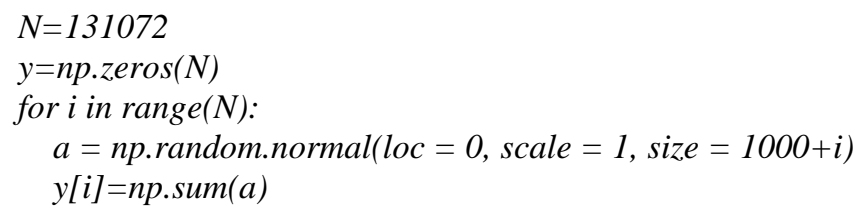

The results of the calculating are shown in Figure 1. The top graph shows the analyzed time series, and the bottom graph represents the points corresponding to the lengths of the flat curve, depending on the time interval $\mathrm{k}$, presented in a double logarithmic scale. The required fractal dimension of the time series is calculated from the angle of inclination of the straight line drawn through these points, taking into account the least-squares method.

It is noteworthy that the algorithm we have implemented for the test suite, implemented by executing the program code presented above, in our opinion, implementing the recommendations proposed by Higuchi, consistently gave a dimension close to 2, while in [1] it is said that the set obtained by a similar algorithm should have a fractal dimension of 1.5. The fact that the expected dimension does not coincide with the calculated one may indicate either that the implementation of the algorithm for calculating the fractal dimension was performed with an error, or that the test array was generated not quite correctly.

Nevertheless, the software implementation of the algorithm for calculating the fractal dimension obtained by us in Python gave stable results for various test sets, which were invariant to a change in the scale of the sample under study, both in time and in amplitude.

Based on this, it was decided to carry out computational experiments using the software version we obtained for calculating the fractal dimension of the time series. 

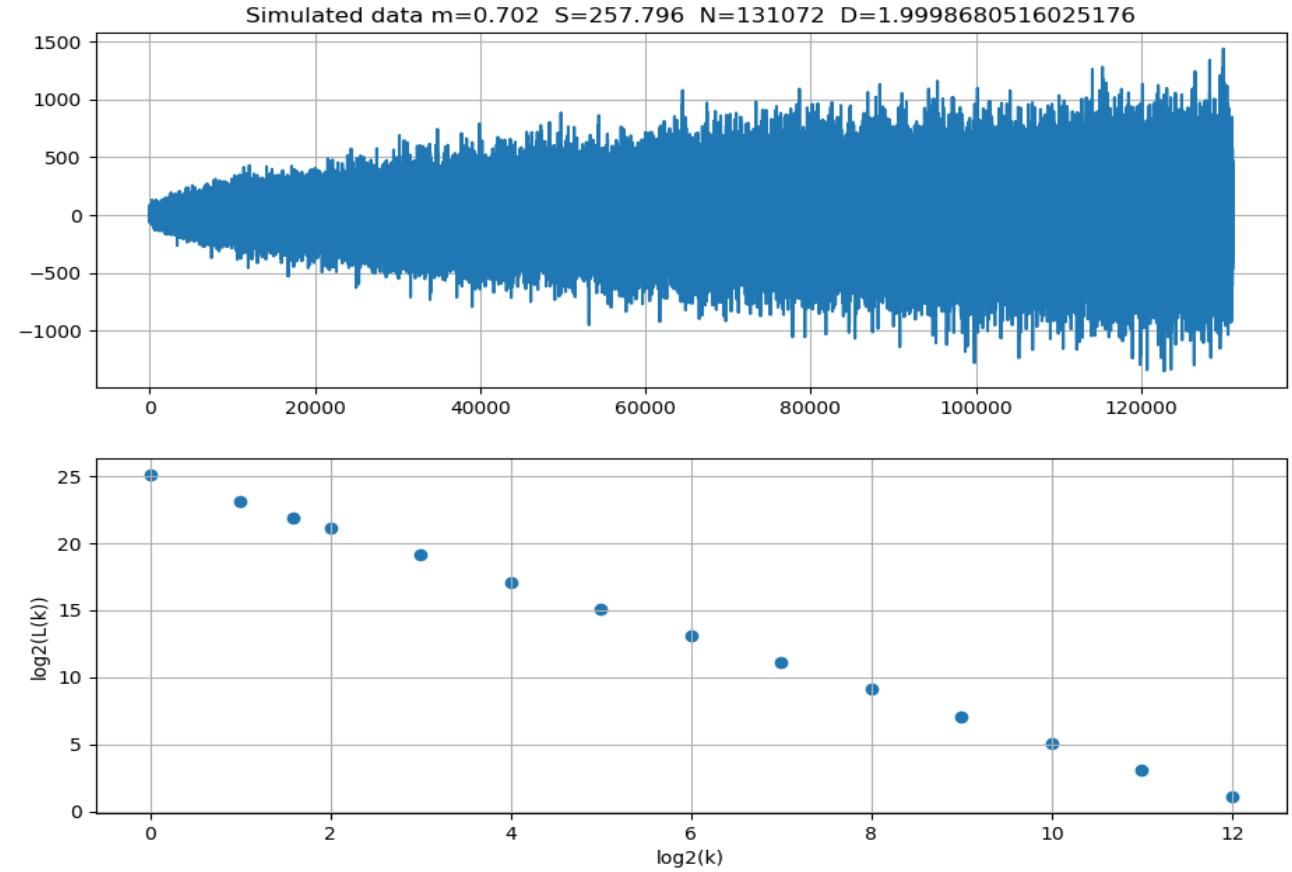

Fig. 1. The modeled time series and points of the straight line, by the slope of which the fractal dimension is calculated.
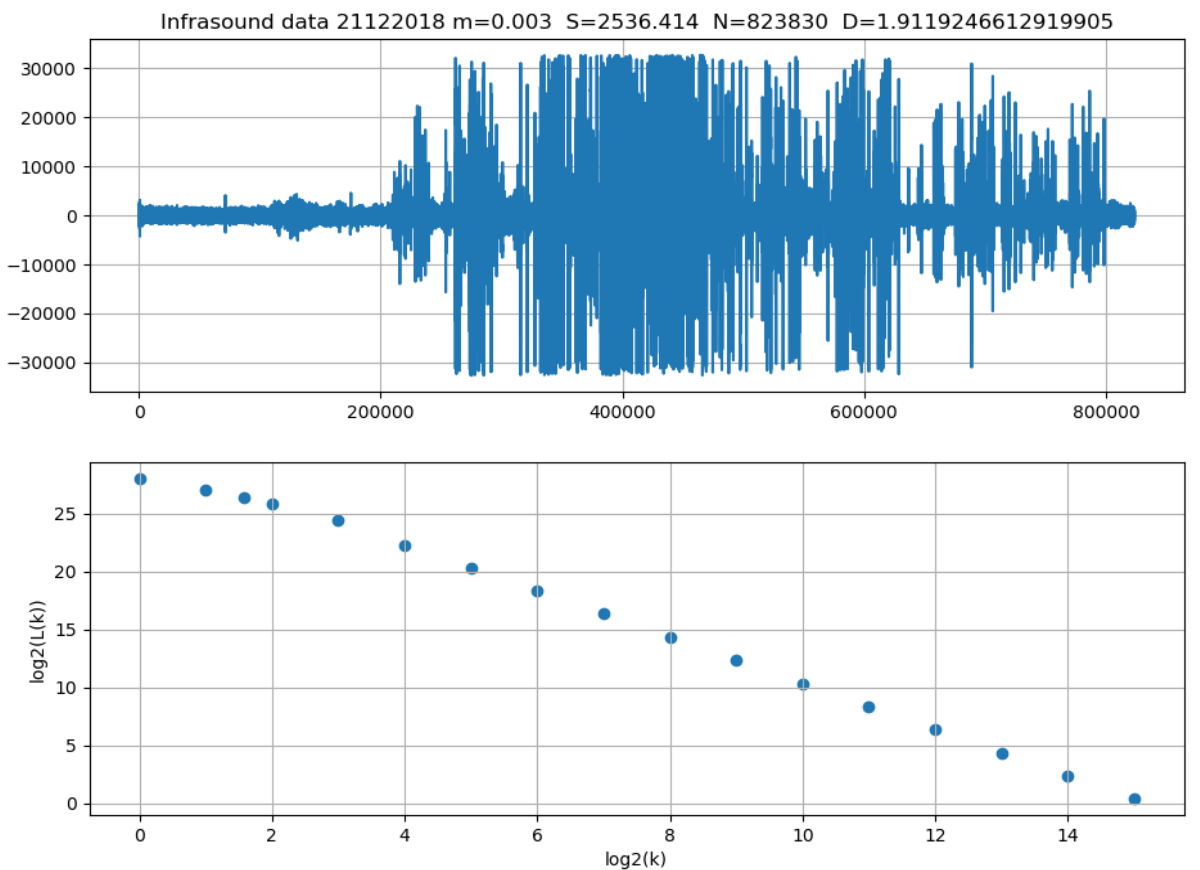

Fig. 2. Time series with an infrasonic 'event'. 
The next step in the computational experiment was devoted to calculating the fractal dimension of the daily time series containing the signal received from the infrasonic 'event'. It should be noted that the calculation of the required fractal dimension gave us nothing.

Visual analysis of fragments of the studied time series showed a significant change in the type of the observed signal during the recording of data from the observed infrasonic 'event'. Based on this, a hypothesis was put forward that the fractal dimensions of the segments of the time series before the observed event and during the observed event should change. As a training sample, we had to select signals of phenomena of sufficient power, for which the time of their appearance would be known exactly. Thus, the daily time series of the days when the Proton rocket was launched from the Baikonur cosmodrome were selected. Infrasonic sensors recorded the moments of separation of the stages of the carrier rocket. Figure 3 shows one of these signals
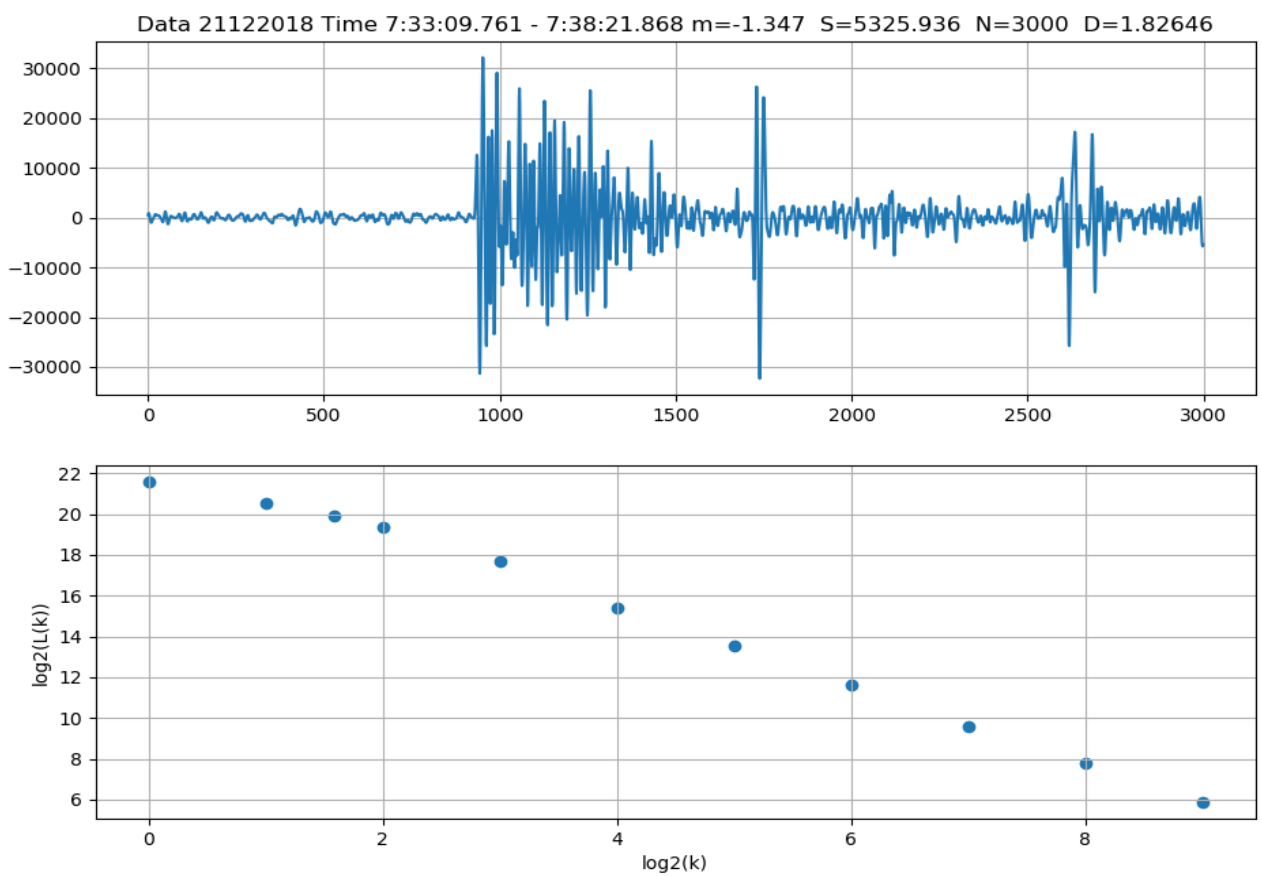

Fig. 3. Signal from the falling stages of the launching rocket Proton.

The computational experiment showed that the fractal dimension of the time series before the moment of fixation of the phenomenon and after the start of fixation of the phenomenon are really different, however, it was necessary to accurately determine the time (ordinal number of counts) that recorded this event. As a result of calculating the fractal dimensions of various fragments of the time series before the 'event', during the 'event' and after it, it was noticed that the values of the fractal dimension of the investigated fragment of the time series, where the beginning of the 'event' falls, noticeably changes. It was empirically determined that a sufficiently distinguishable jump in fractal dimension for signals of different amplitudes can be observed when segmentation of the time series at our disposal with a step of 250 samples. 

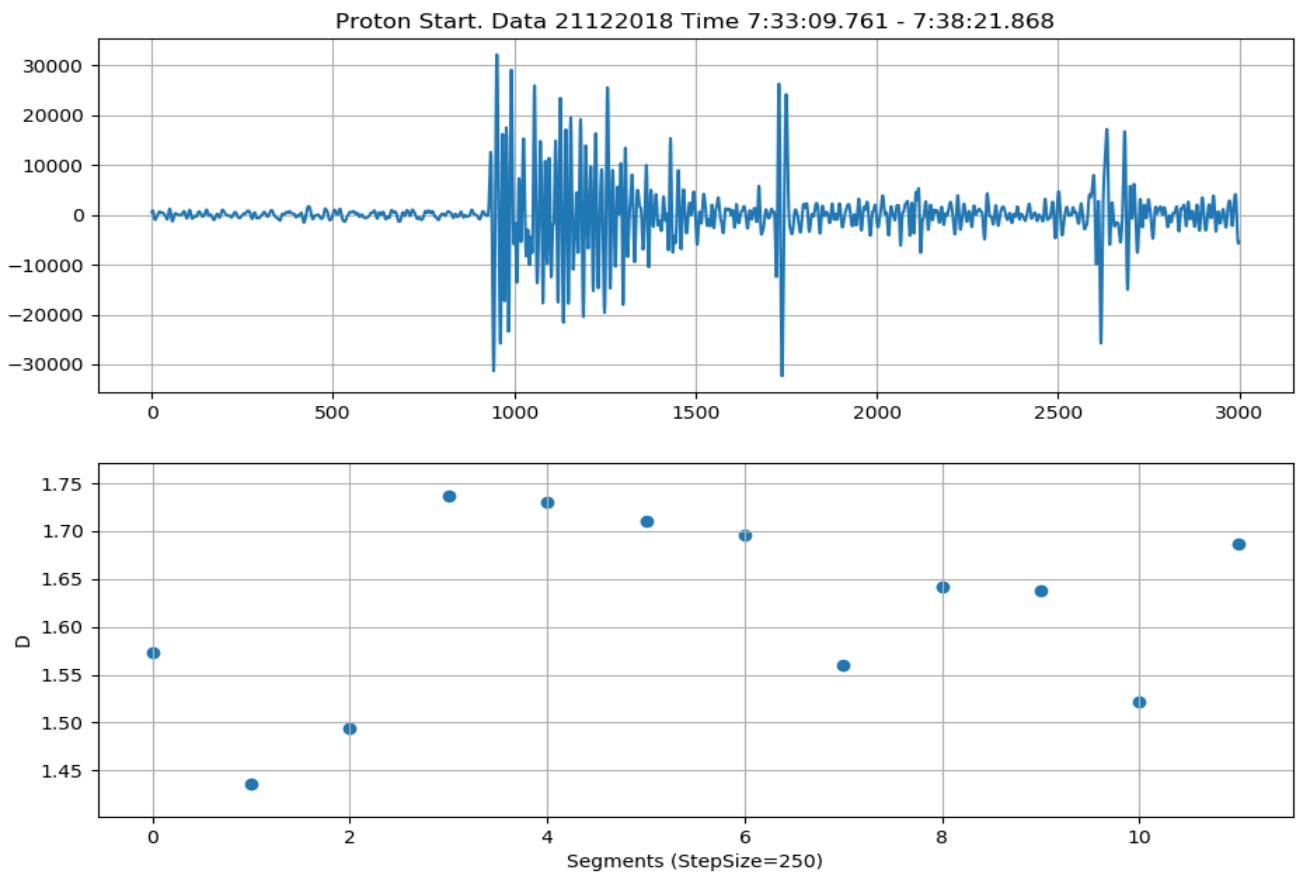

Fig. 4. 'Event' signal and fractal dimensions of time series segments.

In Figure 4, the upper graph shows the signal of the desired infrasonic 'event', and the lower graph shows the values of the fractal dimensions of the corresponding intervals of the time series, segmented with a step of 250 .

It is important to note that the sampling rate of the signal was about $10 \mathrm{~Hz}$, and the period of most of the investigated signals of "events" corresponded to 10 samples, i.e. about $1 \mathrm{~Hz}$. Thus, a segment of a time series could include about 25 signal periods. It is possible that these ratios of the segment length and the period of the sought-for 'event' signal can be useful when working with signals that are excited by phenomena of a different energetic order and, accordingly, having other wavelengths of infrasonic signals.

In the course of analyzing the fractal dimensions of segments of different time series, it was noted that practically every investigated time series contained jumps in dimensions in time intervals, including the desired 'event' and in time intervals different from the presence of the phenomenon under study. Moreover, in such time intervals ('empty segments'), as a rule, various low-amplitude noise signals were presented. In other words, finding the moments of change in the fractal dimension of the segments of the time frame did not lead to an accurate and unique solution to the problem of identifying an 'event'.

As a result of the analysis, a hypothesis was put forward that for the temporary identification of an 'event' it is necessary to use at least two parameters that reinforce each other - fractal and amplitude. So it was decided to conduct an experiment with the calculation of the element-wise product of the components of the vectors of changes in fractal dimensions and changes in the average amplitudes of the investigated time series. 

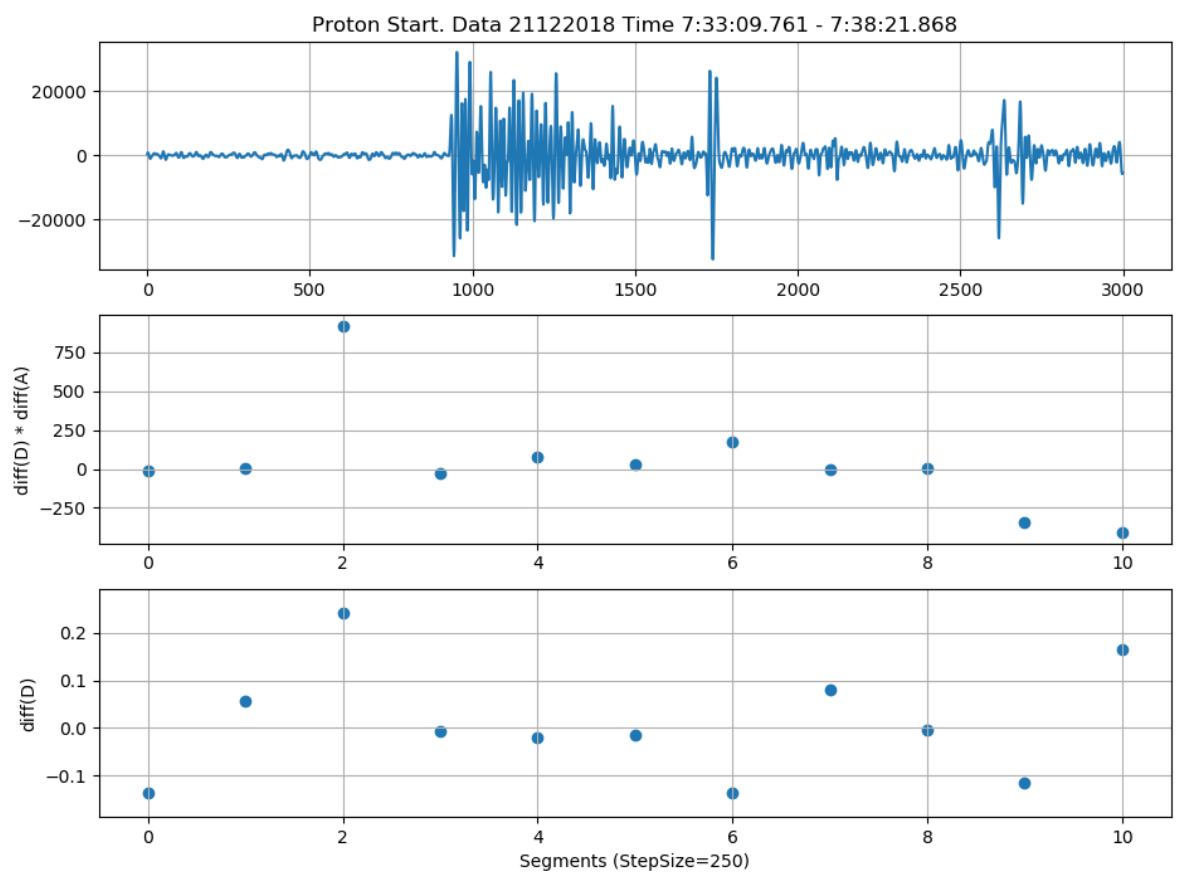

Fig. 5. The result of testing the hypothesis of element-wise multiplication of vectors containing jumps in fractal dimensions and amplitudes jumpings.

To test the hypothesis put forward, not only the fractal dimension, but also the expectation function of the signal amplitude was calculated on segmented time intervals, and then, using the numpy.diff() function, vectors of amplitude and fractal dimensions "jumps" were found. Next, performed element-wise multiplication of the vectors of "jumps" of amplitudes and fractal dimensions. The result was expected and positive. As you can see in Figure 5, the graph of the points of the resulting vector of element-byelement products contains the maximum value corresponding to the time interval of the sought-for 'event' signal.

Figure 6 and Figure 7 (scaled version) show the results of a computational experiment of the signal detecting of the desired infrasonic "event" in daily data.

As seen, visually detecting an 'event' with a graph of daily infrasound monitoring data in front of the eyes is a very problematic task. At the same time, the use of our proposed approach allows us to detect the desired event' with an accuracy of the segment number.

Figures 8 and 9 show the results of a computational experiment to search for an infrasonic 'event' in the time series data from 10/09/2019. 

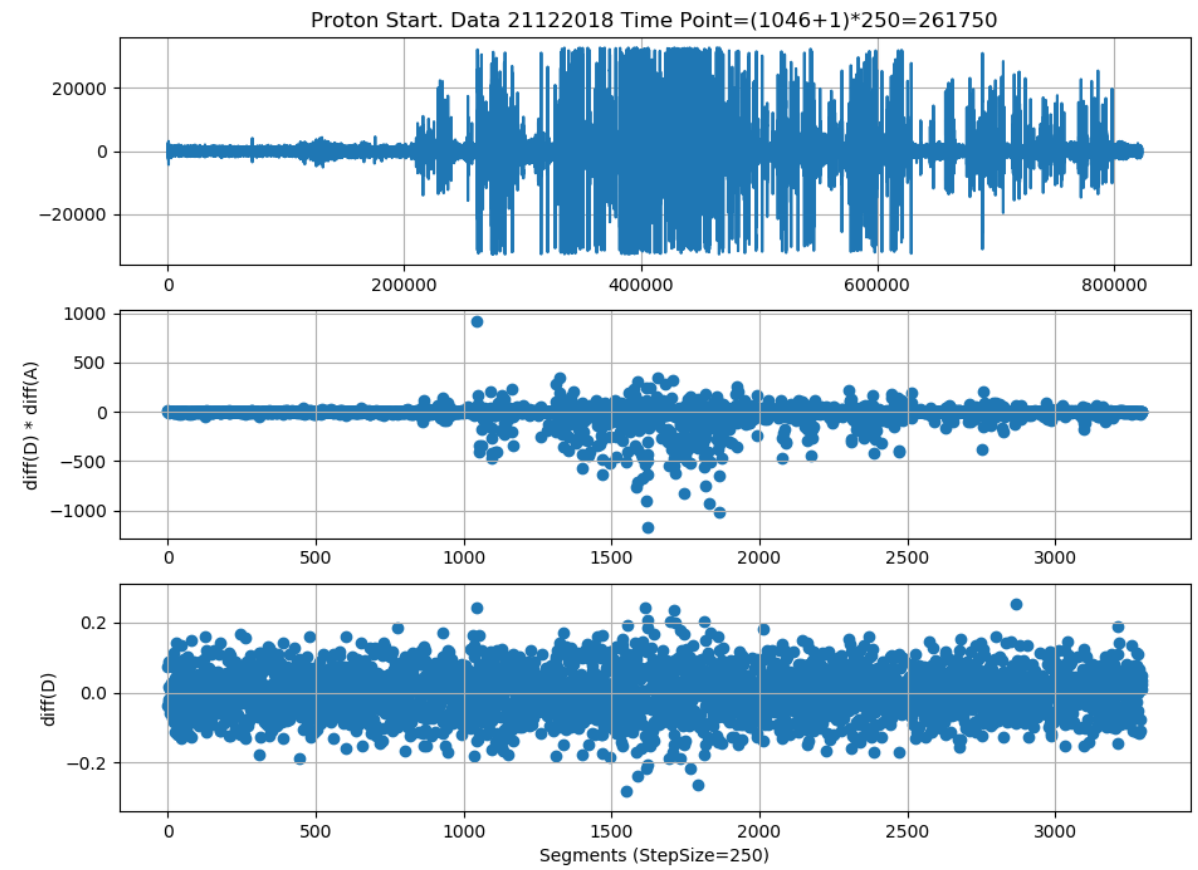

Fig. 6. The results of a computational experiment with a vector of products of fractal dimension differences and amplitude differences for an 'event' dated 12/21/2018.

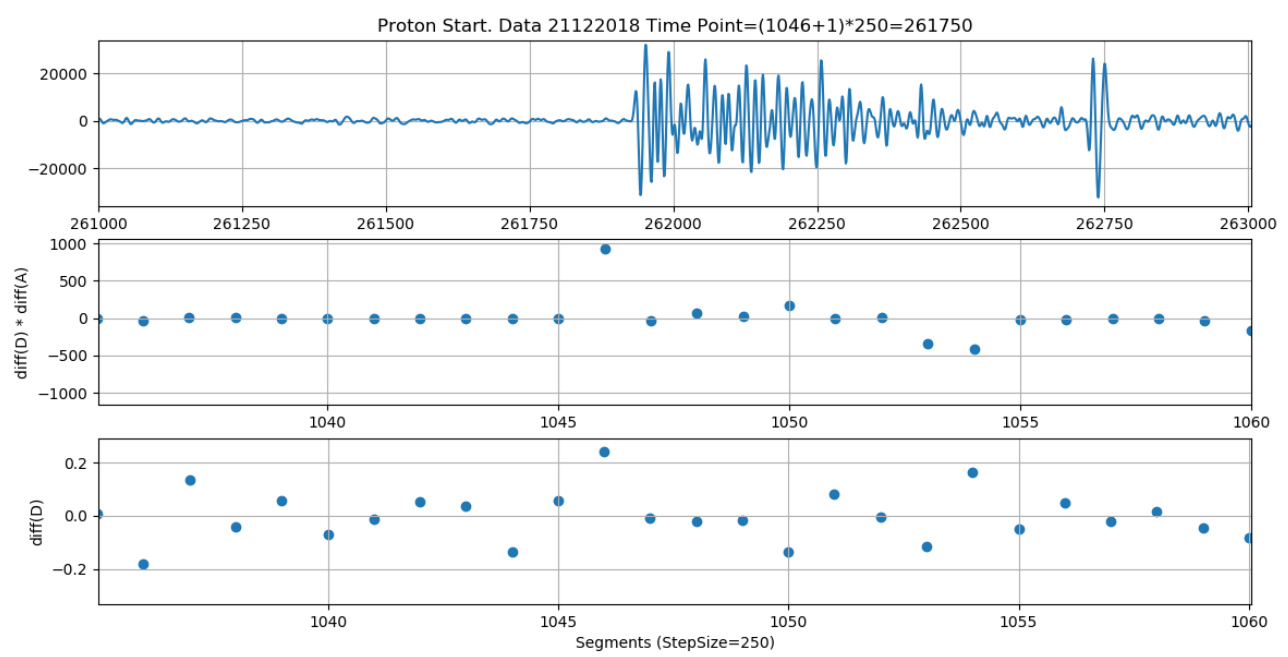

Fig. 7. Results of a computational experiment for a daily time series from 12/21/2018 (scaled). 

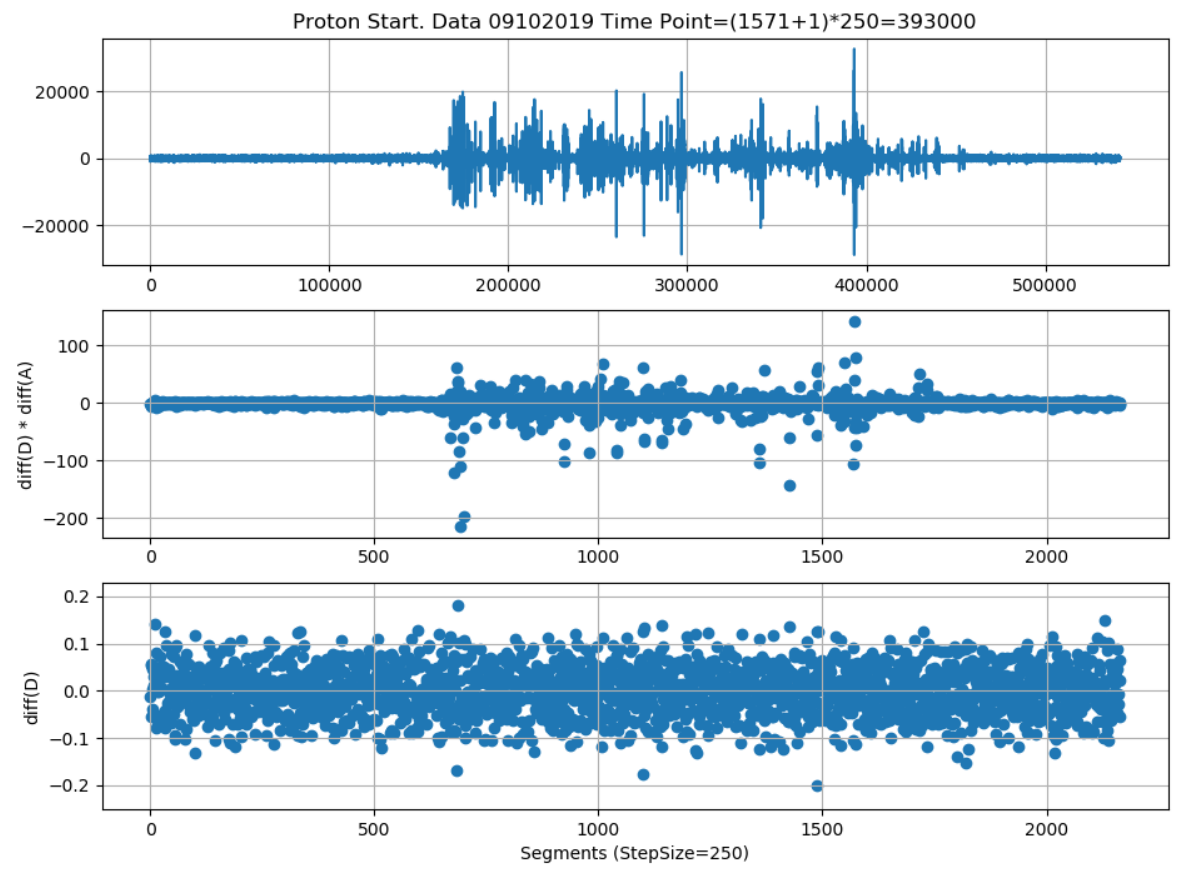

Fig. 8. The results of a computational experiment with a vector of products of fractal dimension differences and amplitude differences for an 'event' dated 10/09/2019.

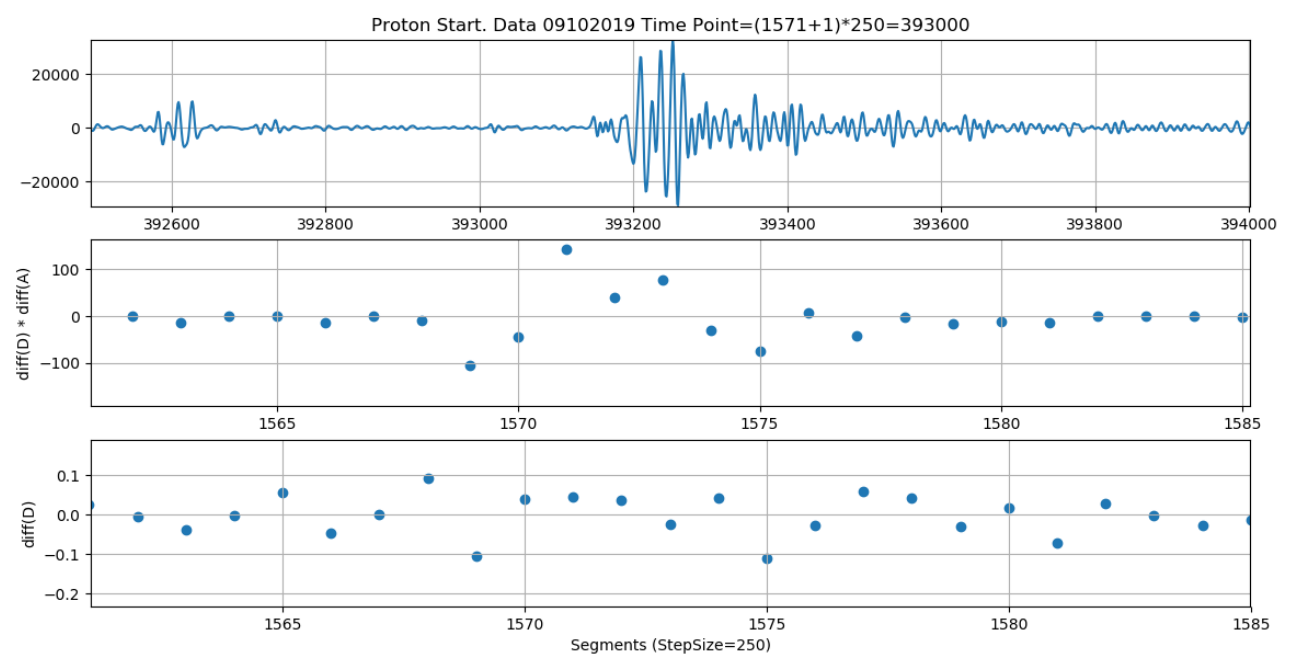

Fig. 9. Results of a computational experiment for a daily time series from 10/09/2019 (scaled).

\section{Conclusion}

In the process of studying the time series of infrasonic signals obtained as a result of measuring experiments, a number of regularities were discovered that make it possible to propose an original heuristic scheme (procedure) for processing and transforming the studying signal. The proposed approach makes it possible to determine the time intervals of 
the fragments of the investigated measurement results corresponding to natural phenomena causing infrasonic waves.

The essence of the used heuristic procedure is to perform the following steps:

- preparation of a time series by processing the data with a simple normalized difference filter, pre-smoothed by performing a coenvolution (convolution) operation with a Gaussian kernel;

- determining the step of segmenting the prepared data array;

- calculating the vector of fractal dimensions and the vector of averaged amplitudes of the time series segments obtained in the previous step;

- calculation of vectors of differences of neighboring values (jumps) for the vector of fractal dimensions and for the vector of averaged amplitudes;

- element-wise multiplication of jump vectors of fractal dimensions and averaged amplitudes;

- finding the maximum values for the resulting vector of element-wise multiplication of jump vectors of fractal dimensions and averaged amplitudes of time series segments.

The timestamps of the maximum values found will correspond to the time intervals of the studied series corresponding to the sought-for irregular natural phenomena that generate infrasonic waves.

The study was carried out with the financial support of the Russian Foundation for Basic Research and the Government of the Altai Republic within the framework of scientific project No. 20-41340003 p_a "Study of effectiveness of the method of project interfaces using when implementing the project approach in the training system for engineering personnel and artificial intelligence specialists in the Altai Republic".

\section{References}

1. T. Higuchi, Physica D: Nonlinear Phenomena, 31(2), 277, (1988)

2. P. Powell, European Journal of Engineering Education, 29(2), 221, (2004)

3. V.S. Gruzinov, Izvestia vuzov «Geodesy and Aerophotosurveying», 63(1), 45, (2019)

4. D. Roberts, E. Bradley, K. Roth, T. Eckmann, C. S. Linking, Journal of Geoscience Education, 58(5), 262, (2010)

5. S. Rohini, S. Agrawal, IEEE International Conference In Communication and Signal Processing (ICCSP), 2008, (2016)

6. X. Liu, O. Baiocchi, 7th IEEE Conference In Information Technology, Electronics and Mobile Communication (IEMCON), 1, (2016)

7. N. Kudryavtsev, A. Temerbekova, Novosibirsk State Pedagogical University Bulletin, 8(6), 167, (2018)

8. N.G. Kudryavtsev, A.Yu. Gozdarev, D.V. Kudin, E.O. Uchaikin, A.A. Temerbekova, Journal of Physics: Conference Series Computer-Aided Technologies in Applied Mathematics, 1680, 012030, (2020)

9. F.C.D. la Torre, J.I. Gonzalez-Trejo, C.A. Real-Ramirez, L.F. Hoyos-Reyes, Journal of Physics: Conference Series, 475, 1, (2013)

10. S. Spasic, Chaos, Solitons \& Fractals, 69, 179, (2014)

11. C. Bandt, Ecological Modelling, 182(3-4), 229, (2005)

12. C. Bandt, and B. Pompe, Phys. Rev. Lett., 88, 174102, (2002)

13. R. Esteller, G. Vachtsevanos, J. Echauz, B. Litt, IEEE Transactions on Circuits and Systems I: Fundamental Theory and Applications, 48(2), 177, (2001)

14. F.L. Giudicepietro, C.G. Macedonio, et al, Sci Rep, 10, 1, (2020) 
15. J. Krzyszczak, P. Baranowski, M. Zubik, et al, Theor Appl Climatol, 137, 1811, (2019)

16. L.J.X. Zhang, J.Z. Gong, J.T. Tang, Z.Y. Ren, L.G, Deng, J. Cai, Fractals, 26(2), 1840011, (2018)

17. D. Nikolopoulos, E. Petraki, P.H. Yannakopoulos, G. Priniotakis, I. Voyiatzis, D. Cantzos, Geosciences, 10(6), 1, (2020) 